\title{
RBF Neural Network of Sliding Mode Control for Time-Varying 2-DOF Parallel Manipulator System
}

\author{
Haizhong Chen and Songlin Wo \\ Electrical and Information Engineering institute, Jiangsu University of Technology, Changzhou, Jiangsu, 213001, China \\ Correspondence should be addressed to Songlin Wo; wosonglin2000@yahoo.com.cn
}

Received 17 September 2013; Revised 16 October 2013; Accepted 16 October 2013

Academic Editor: Rongni Yang

Copyright (C) 2013 H. Chen and S. Wo. This is an open access article distributed under the Creative Commons Attribution License, which permits unrestricted use, distribution, and reproduction in any medium, provided the original work is properly cited.

\begin{abstract}
This paper presents a radial basis function (RBF) neural network control scheme for manipulators with actuator nonlinearities. The control scheme consists of a time-varying sliding mode control (TVSMC) and an RBF neural network compensator. Since the actuator nonlinearities are usually included in the manipulator driving motor, a compensator using RBF network is proposed to estimate the actuator nonlinearities and their upper boundaries. Subsequently, an RBF neural network controller that requires neither the evaluation of off-line dynamical model nor the time-consuming training process is given. In addition, Barbalat Lemma is introduced to help prove the stability of the closed control system. Considering the SMC controller and the RBF network compensator as the whole control scheme, the closed-loop system is proved to be uniformly ultimately bounded. The whole scheme provides a general procedure to control the manipulators with actuator nonlinearities. Simulation results verify the effectiveness of the designed scheme and the theoretical discussion.
\end{abstract}

\section{Introduction}

The past several decades have seen a rapid increase in parallel manipulators connected to the control system. It is well known that parallel manipulators with high rigidity are of generally higher accuracy and of lower error accumulation than similarly sized serial manipulators. Their closed kinematics structure allows them to obtain high structural stiffness and perform high-speed motions. The inertia of its mobile parts is reduced, since the actuators of a parallel manipulator are often fixed to its base and the end effectors can perform movements with higher accelerations. Adaptive tracking control for a class of nonlinear systems is given in [1-3], and in [4] a design of the sliding mode surface integral algorithm is proposed to inhibit the steady-state error and enhance the robustness. In $[5,6]$ sliding mode control (SMC) with low-pass filter is used to keep trajectory tracking accurately.

It is known that SMC has the intrinsic nature of robustness, good transient fast response, and insensitivity to the variation of plant parameters and external disturbances in [7]. Thus, the SMC is considered as an effective approach for the control of many systems such as uncertain nonlinear systems in [8], discrete-time nonlinear systems in [9], and singular stochastic hybrid systems in [10-12]. The control process of SMC consists of two parts; one part is continuous and the other discontinuous. When the system reaches the sliding mode, the system with variable structure control is insensitive to the external disturbances and the variations of the plant parameters, and it has been widely applied to the manipulator system due to its operation characteristics for the sake of fastness, robustness, and stability in large load variations. All those merits are gotten at the cost of the chattering. Furthermore, in many time-variable systems, parameters and perturbation upper bounds are often uncertain, so switching control gain should be as high as possible to keep stable [1316]. The control algorithm in [17-20] reduces the reaching phase extremely and achieves better robustness than SMC. However, in [17] with disturbance observer based on SMC, the algorithm assumes that the disturbance is produced by a linear exogenous system, but in fact it is difficult to accurately predict the uncertainty and disturbance of the time-variable system. In [18-20], the adaptive sliding mode control (ASMC) algorithm is proposed with the switching 


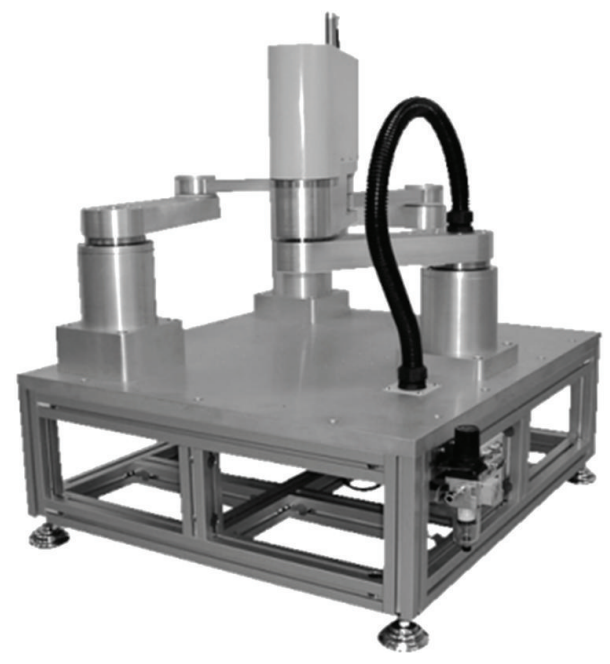

FIGURE 1: Structure of 2-DOF parallel manipulator.

gain excessive the SMC values, which brings the more serious chattering problems. So the appropriate switching gain is the key to the accurate control in time-variable system.

Motivated by the above observations, we propose a new approach to using RBF neural network to estimate the actuator nonlinearities and their upper boundaries for the switching gain. In this paper, we aim to solve these problems by focusing on the accurate tracking problem of the uncertain mechanical system. Our research begins with a time-variable SMC (TVSMC) algorithm with the dynamics of the manipulator. The main characteristics of such a TVSMC algorithm are discussed. To deal with the finite static error brought by the continuous approximation of the TVSMC algorithm, the RBF neural network is utilized, a novel Lyapunov function is introduced, and then a new time-variable stability criterion is presented. In the numerical simulation, the RBF TVSMC algorithm and the TVSMC algorithm are compared after being tested.

\section{System Model}

The 2-degree-of-freedom parallel manipulator (2-DOF parallel manipulator) is made up of three groups of two links in one platform, in which one group has a base of open chain mechanism, respectively, installed by the AC servo motor and a speed reducer drive shown in Figure 1 and coordinate in Figure 2. The 2-DOF parallel manipulator system can be described by Laugrange's equations [21, 22]:

$$
J \dddot{\theta}+B \ddot{\theta}+W \dot{\theta}=U+U_{d} .
$$

In (1), $\theta \in R^{3}$ stands for a displacement angle of generalized coordinates, $J$ for the symmetric and positivedefinite inertia matrix, $B$ for a damping coefficient matrix, $W$ for a stiffness coefficient matrix, $U$ for a voltage vector of generalized control input, and $U_{d}$ for a damping voltage vector of external disturbance.

Defining $J=\widehat{J}+\Delta J, B=\widehat{B}+\Delta B$, and $W=\widehat{W}+\Delta W$, the superscript $(\wedge)$ stands for the nominal value, and the notation

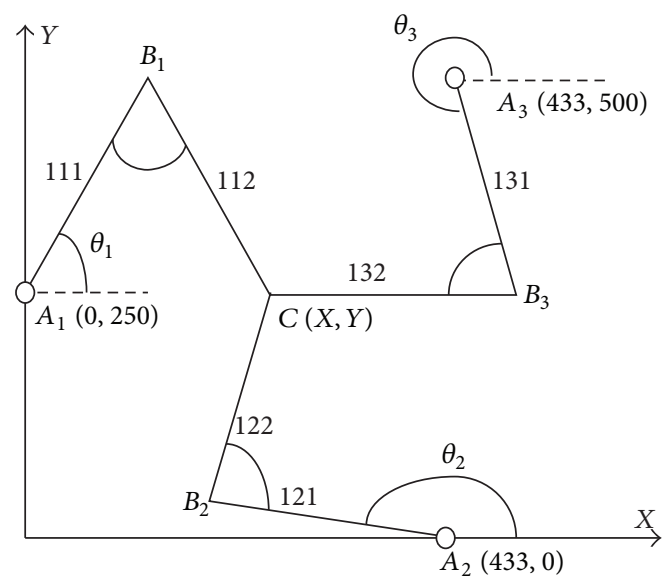

FIgURE 2: Coordinate of 2-DOF parallel manipulator.

$(\Delta)$ represents the uncertainty. According to the structural feature, the effect caused by the model uncertainties can be merged into the disturbance term, which then can be regarded as the lumped disturbance in the following form:

$$
d=U_{d}-\Delta W \dot{\theta}-\Delta B \ddot{\theta}-\Delta J \ddot{\theta} .
$$

From (2), it is assumed that the lumped disturbance is bounded by a upper bound; that is, $\|d\|_{\infty} \leq d_{\max }$, where $d_{\max } \in R^{+}$is a constant scalar and $\|\cdot\|_{\infty}$ is the infinite norm of a vector. $\dot{\vec{J}}-2 \widehat{B}$ is a skew-symmetric matrix (see details in $[23,24])$.

So (1) can be rewritten as

$$
\widehat{J} \ddot{\theta}+\widehat{B} \ddot{\theta}+\widehat{W} \dot{\theta}=U+d .
$$

\section{TVSMC Design and Parameter Setting}

In this paper, we will address the tracking control problem of the system in (3). For the desired trajectory with the system states of $\theta_{d}, \dot{\theta}_{d}$, and $\ddot{\theta}_{d}$, a controller $u$ for the system is designed so that the system states $\theta_{d}, \dot{\theta}_{d}$, and $\ddot{\theta}_{d}$ can track the desired trajectory in the presence of parametric uncertainty and external disturbance.

For the given trajectory, the tracking error and tracking angle are defined as

$$
\begin{aligned}
& E=\theta-\theta_{d}, \\
& \dot{E}=\dot{\theta}-\dot{\theta}_{d}, \\
& \ddot{E}=\ddot{\theta}-\ddot{\theta}_{d},
\end{aligned}
$$

with the initial tracking error satisfying $E(0) \neq 0_{3 \times 1}$, $\dot{E}(0) \neq 0_{3 \times 1}$, and $\ddot{E}(0) \neq 0_{3 \times 1}$, where the subscript denotes the appropriate dimensions of the matrix. 


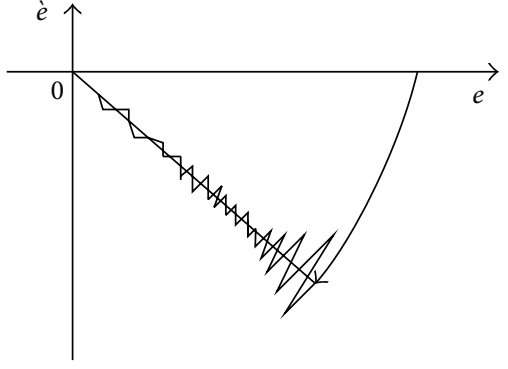

(a) Phase locus

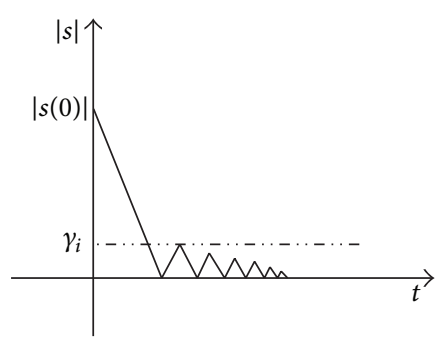

(b) Sliding mode function

Figure 3: Phase locus and sliding mode function.

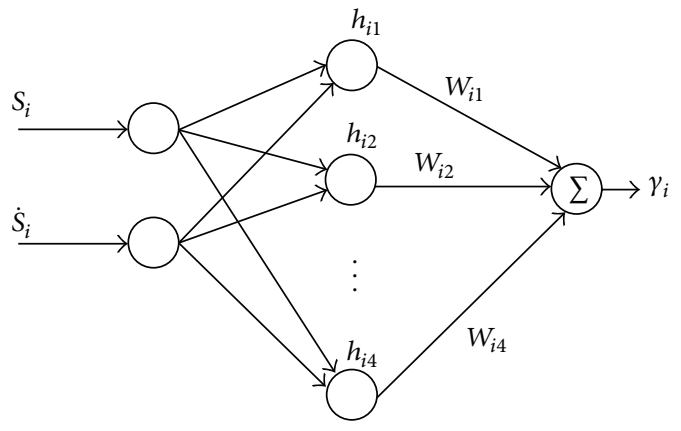

FIGURE 4: Architecture of the RBF neural network.

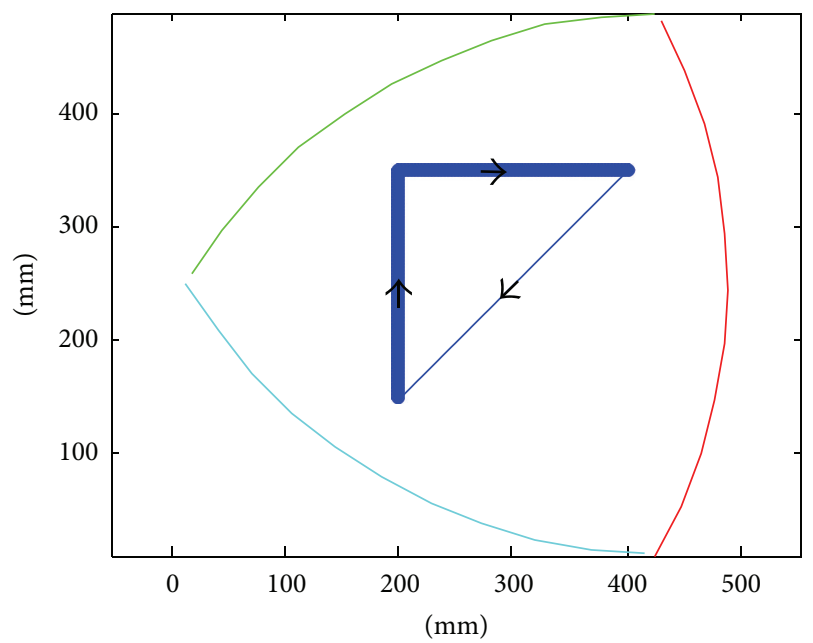

FIGURE 5: The desired manipulator trajectory.

In order to derive the SMC algorithm, the switching surface can be chosen as

$$
S=\left[\begin{array}{lll}
s_{1} & s_{2} & s_{3}
\end{array}\right]^{T}=C E,
$$

where $C \in R^{3 \times 3}$ is the matrix with strictly positive every elements and $E=\left(\begin{array}{lll}E & \dot{E} & \ddot{E}\end{array}\right)^{T}$. The switching surface is then determined by $S=0_{3 \times 1}$, which is the desired dynamics.

The input of SMC algorithm is set as

$$
U=u_{\text {eq }}+u_{n} \text {. }
$$

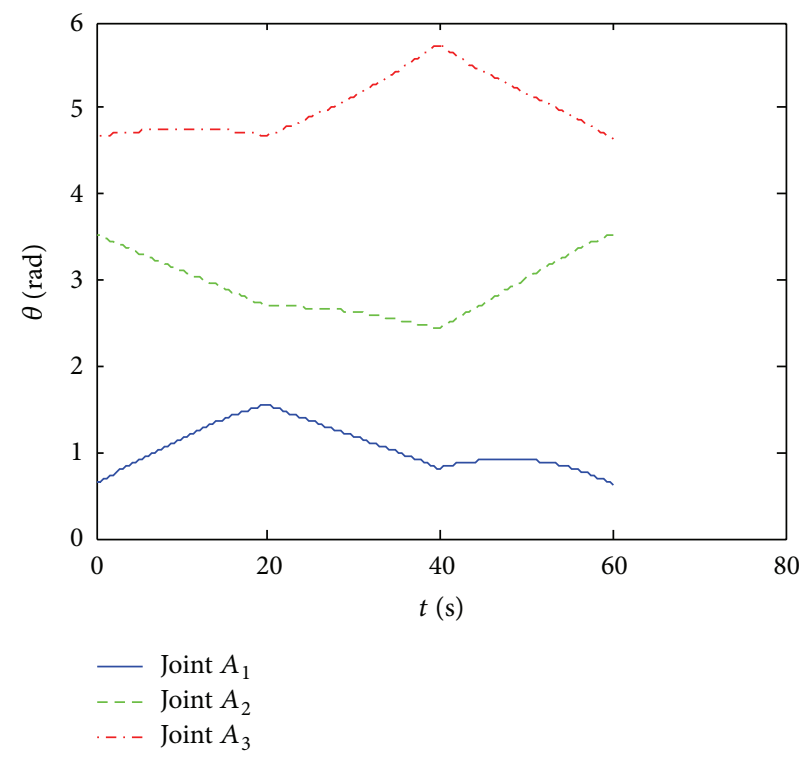

Figure 6: The desired joints trajectories.

In (6), $u_{\mathrm{eq}}$ is the equivalent control. The equivalent control of the ideal sliding mode is obtained on $S=0$ with $\dot{S}=0$; it can be gotten as

$$
\begin{gathered}
u_{\mathrm{eq}}=-\left(\widehat{J}\left(C_{1} \dot{E}+C_{2} \ddot{E}\right)-\widehat{B}\left(C_{1} E+C_{2} \dot{E}\right)\right. \\
\left.+\left(\widehat{J} \dddot{\theta}_{d}+\widehat{B} \ddot{\theta}_{d}+\widehat{W} \dot{\theta}_{d}\right)\right) .
\end{gathered}
$$

$u_{n}$ is the switching control:

$$
u_{n}=-\gamma \cdot \operatorname{sign}(S) \text {. }
$$

$\gamma=\operatorname{diag}\left(\gamma_{1}, \gamma_{2}, \gamma_{3}\right) \in R^{3 \times 3}$ is the switching gain matrix with the elements $\gamma_{i}>d_{\max }$, and the $\operatorname{sign}(S)=$ $\left(\operatorname{sign}\left(s_{1}\right) \operatorname{sign}\left(s_{2}\right) \operatorname{sign}\left(s_{3}\right)\right)^{T}$ presents the sign function.

Theorem 1. Considering the system in (3) under the lumped disturbance with an upper boundary of $d_{\max }$, by adopting the time-varying sliding mode function in (5) and the corresponding input control in (6), if $\gamma_{i}>d_{\max }$, then the controlled system is stable. 


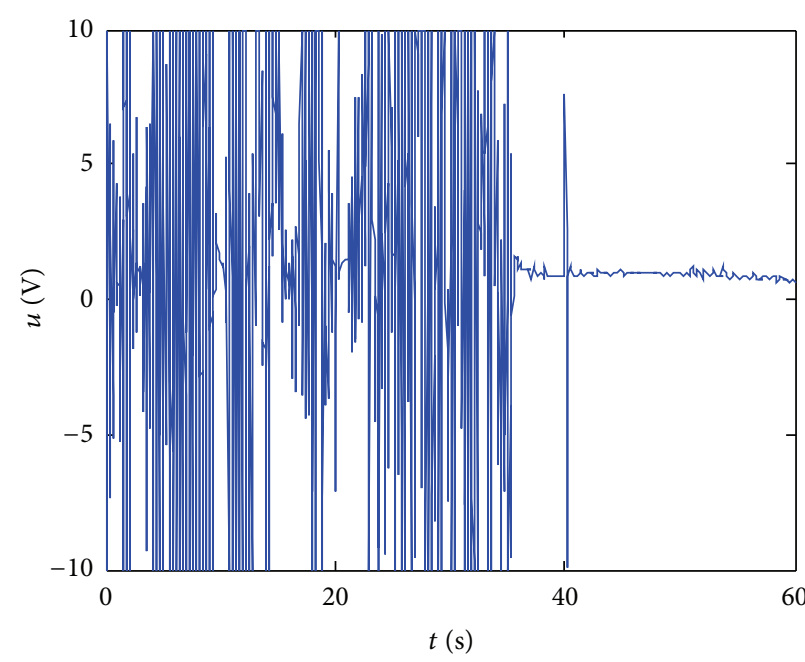

(a) Control voltage of joint $A_{1}$

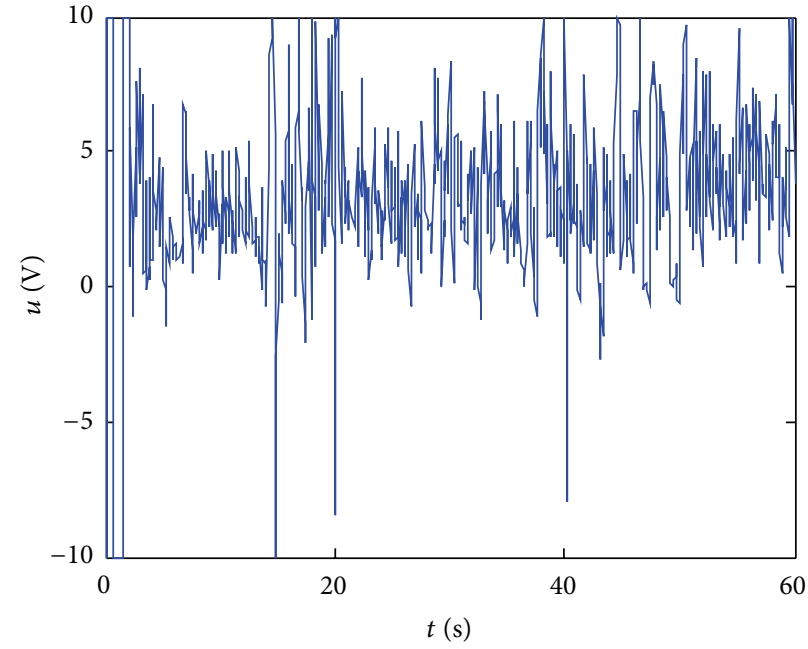

(b) Control voltage of joint $A_{2}$

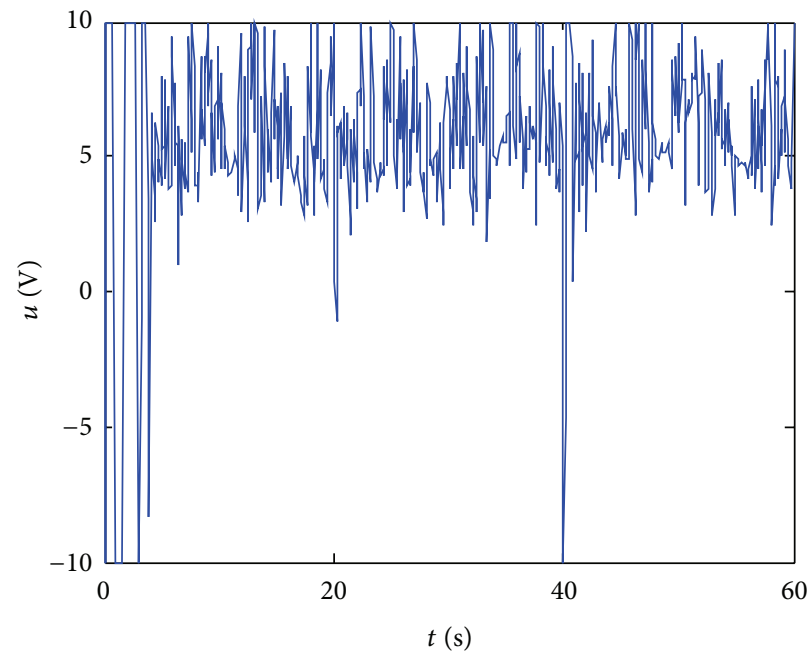

(c) Control voltage of joint $A_{3}$

FIGURE 7: Control voltage generated by TVSMC.

Proof. Consider the following Lyapunov function:

$$
V=\frac{1}{2} S^{T} \widehat{J} S
$$

Differentiating $V$ with respect to time yields

$$
\begin{aligned}
\dot{V}= & S^{T} \widehat{J} \dot{S}+\frac{1}{2} S^{T} \dot{\widehat{J}} S \\
= & S^{T} \widehat{J}\left(C_{1} \dot{E}+C_{2} \ddot{E}+\ddot{E}\right)+\frac{1}{2} S^{T} \dot{\hat{J}} S \\
= & S^{T}\left(U+d+\widehat{J}\left(C_{1} \dot{E}+C_{2} \ddot{E}\right)\right. \\
& \left.\quad-\widehat{B} \ddot{E}-\widehat{W} \dot{E}-\widehat{J}_{d}-\widehat{B} \ddot{\theta}_{d}-\widehat{W} \dot{\theta}_{d}\right) \\
& +\frac{1}{2} S^{T} \dot{\widehat{J}}\left(C_{1} E+C_{2} \dot{E}+\ddot{E}\right)
\end{aligned}
$$

$$
\begin{aligned}
=S^{T}(U & +d+\widehat{J}\left(C_{1} \dot{E}+C_{2} \ddot{E}\right)+\widehat{B}\left(C_{1} E+C_{2} \dot{E}\right) \\
& \left.-\left(\widehat{J} \dddot{\theta}_{d}+\widehat{B} \ddot{\theta}_{d}+\widehat{W} \dot{\theta}_{d}\right)\right) .
\end{aligned}
$$

$U=u_{\text {eq }}+u_{n}$ is substituted in Lyapunov function, and for $\dot{J}-2 B$ is a skew-symmetric matrix such that $(1 / 2) S^{T}(\dot{\vec{J}}-$ $2 \widehat{B}) S=0$. The time derivative of $V$ will be

$$
\begin{aligned}
\dot{V} & =S^{T}(-\gamma \operatorname{sign}(S)+d) \\
& =\sum_{i=1}^{3}\left(-\gamma_{i} \cdot\left|s_{i}\right|+d_{i} s_{i}\right) \\
& \leq-\sum_{i=1}^{3}\left(\gamma_{i}-d_{\max }\right)\left|s_{i}\right|<0 .
\end{aligned}
$$




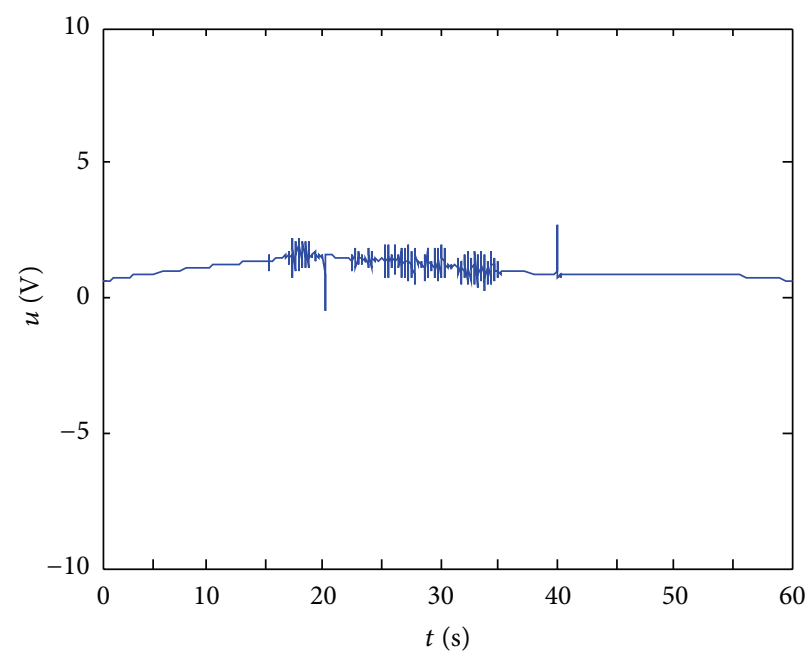

(a) Control voltage of joint $A_{1}$

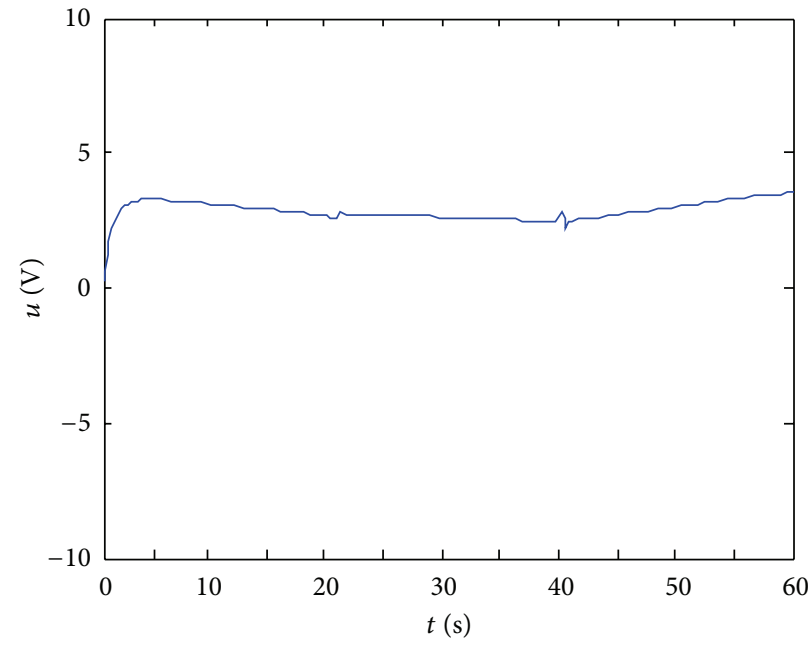

(b) Control voltage of joint $A_{2}$

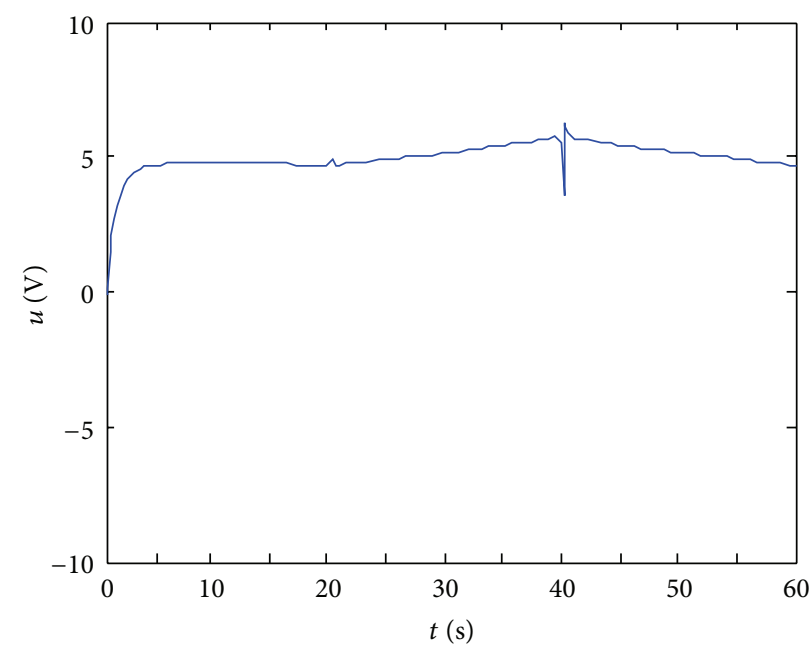

(c) Control voltage of joint $A_{3}$

FIgURE 8: Control voltage generated by RBF TVSMC.

As $V$ is positively defined and $\dot{V}<0$, according to the Lyapunov stability theory, the TVSMC is stable. And Figure 3 is gotten.

\section{RBF SMC Algorithm Design}

From Figure 3(b), the process of SMC action is divided into two states: one is the reaching state and the other is the sliding state. Then reaching state will be joined to the sliding state inside the sliding tranche with bandwidth $2 \gamma$ surrounding the sliding surface, in a finite time limited by the switching frequency. The system adopts the dynamic of the surface and reaches the equilibrium point. In a short phrase, the switching gain depends on $\gamma$. Consider the following saturation function to replace the sign function to decrease chattering:

$$
\operatorname{sat}(S)= \begin{cases}\frac{S}{|S|_{\infty}+\delta}, & \left|s_{i}\right| \leq \delta, \\ \operatorname{sign}(S), & \text { otherwise. }\end{cases}
$$

In (12) $\delta$ is tiny positive number and is also the boundary layer thickness, which can reduce the chattering if appropriately chosen.

On the other hand, radial basis function neural network based on controller design is one of the popular methods of high precision control. In the SMC based controller, the RBF $\mathrm{NN}$ is used to approximate the upper boundary of lumped disturbance (Figure 4).

The upper boundary of the lumped disturbance can be designed as

$$
\gamma_{i}=\sum_{j=1}^{4} w_{i j} h_{i j}
$$

where $h_{i j}$ is the Gaussian radial basis function.

Here, further consideration will be given about adjusting the network weights. The weight adjustment is $A_{d}=$ $(1 / 2) E^{T} E$. 


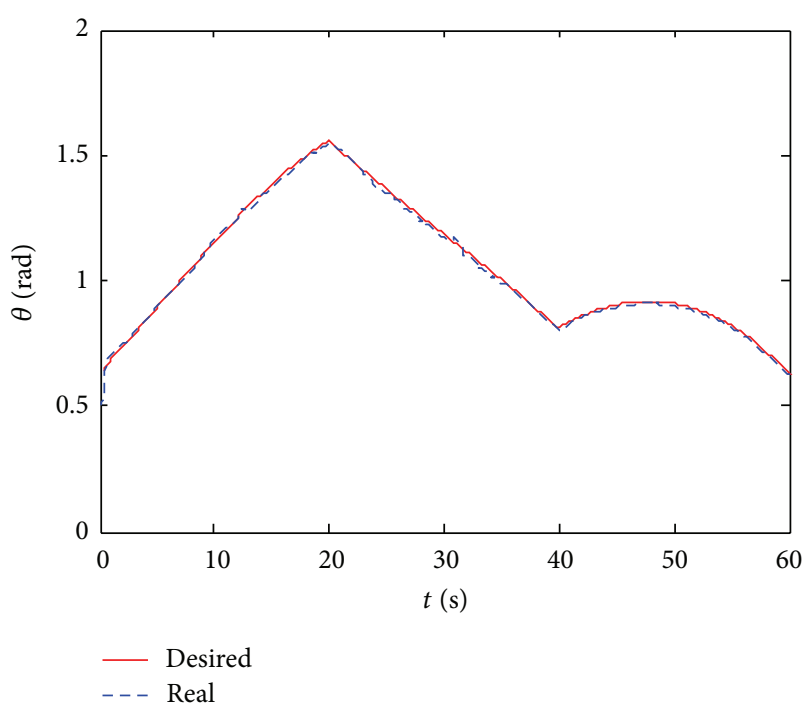

(a) Trajectory tracking responses of joint $A_{1}$

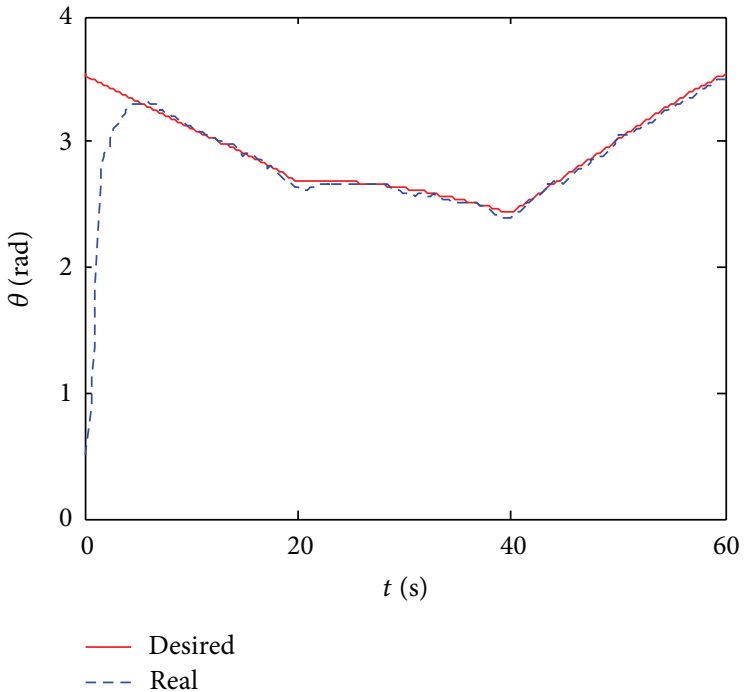

(b) Trajectory tracking responses of joint $A_{2}$

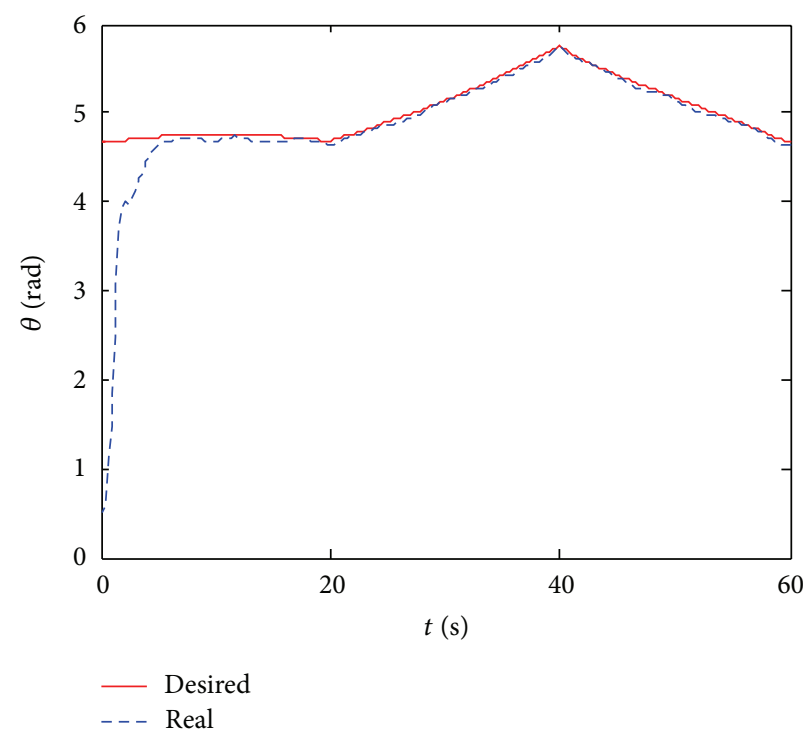

(c) Trajectory tracking responses of joint $A_{3}$

FIGURE 9: Trajectory tracking by TVSMC.

The RBF NN earning algorithm is

$$
\begin{aligned}
\Delta w & =-\frac{\partial A_{d}}{\partial w}=-E^{T} \frac{\partial E}{\partial w} \\
& =-E^{T} \frac{\partial E}{\partial u} \frac{\partial u}{\partial \gamma} \frac{\partial \gamma}{\partial W} \\
& \approx-E^{T} \operatorname{sign}\left(\frac{\partial \theta}{\partial u}\right) \frac{\partial u}{\partial \gamma} \frac{\partial \gamma}{\partial W}
\end{aligned}
$$

where the value of $(\partial \theta / \partial u)$ can be substituted by coefficient of learning rate, $\theta$ is in direct proportion to $u$ in step response, $\operatorname{sign}(\partial \theta / \partial u)=1,(\partial u / \partial \gamma)=-\operatorname{sign}(S)$, and $(\partial \gamma / \partial W)=\phi$.
So $\Delta w$ can be described as follows:

$$
\Delta w \approx E^{T} \operatorname{sign}(S) \phi=\left(C^{-1}\right)^{T}\left|S^{T}\right| \cdot \phi
$$

Theorem 2. Considering the system in (3) under the lumped disturbance with an upper boundary of $d_{\max }$, by adopting the time-varying sliding mode function in (5) and the lumped disturbance identified by using the RBF neural network in (13), the controlled system is global asymptotic stable.

Proof. Let optimal network weights be $w^{*}$ and the estimated upper boundary value of RBF NN be $\tilde{\gamma}$; then we get

$$
\sum_{j=1}^{3} w_{j_{i}}^{*} h_{j}-\tilde{\gamma}_{i}=\varepsilon, \quad|\varepsilon|<\varepsilon_{0},
$$




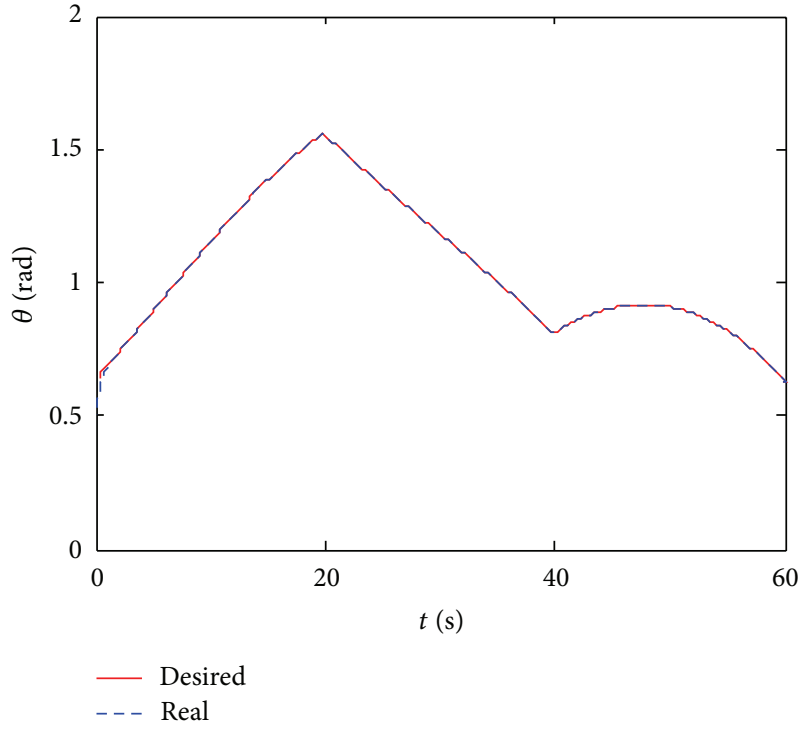

(a) Trajectory tracking responses of joint $A_{1}$

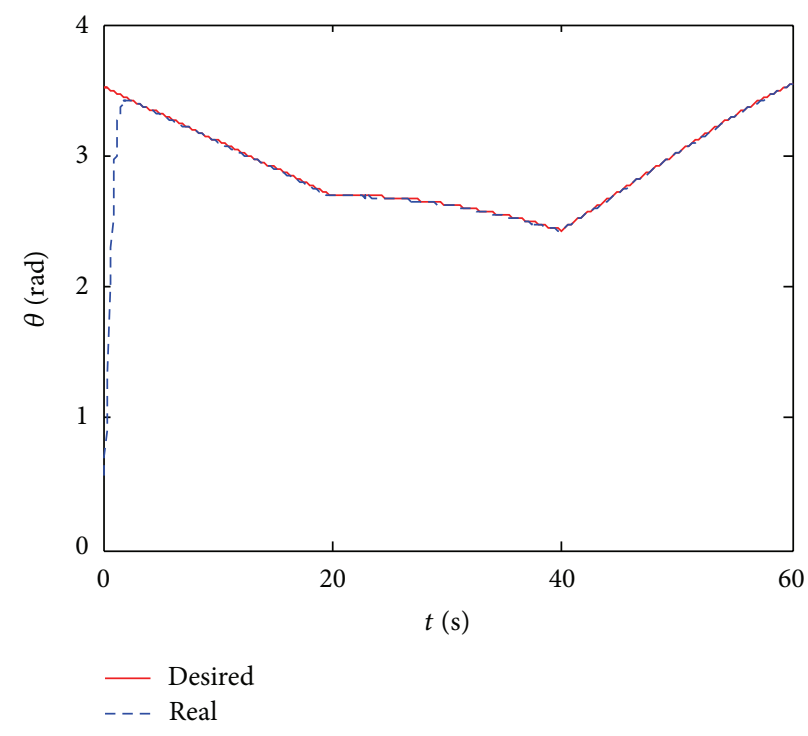

(b) Trajectory tracking responses of joint $A_{2}$

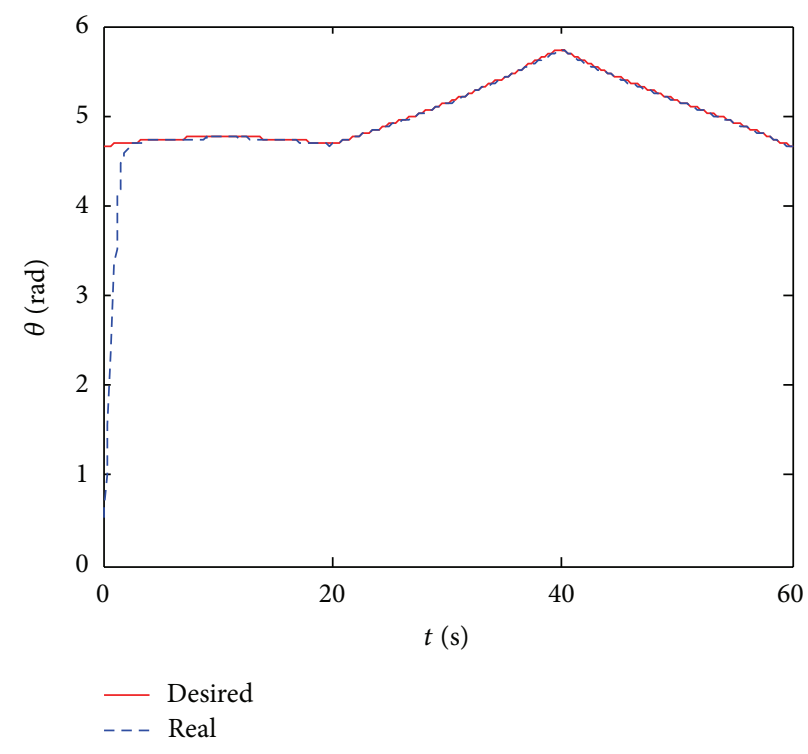

(c) Trajectory tracking responses of joint $A_{3}$

FIGURE 10: Trajectory tracking by RBF TVSMC.

where $\varepsilon$ is a tiny number and $\varepsilon_{0}$ is tiny positive number, and let $\widetilde{\gamma}_{i}-\left|d_{i}\right|>\varepsilon_{1}>\varepsilon_{0}, \dot{w}=\left(C^{-1}\right)^{T}\left|S^{T}\right| \phi$. A neural Lyapunov function can be designed as

$$
V_{1}=\frac{1}{2} S^{T} \widehat{J} S+\frac{1}{2}\left(w^{*}-w\right)^{T} C^{T}\left(w^{*}-w\right) .
$$

Differentiating $V_{1}$ with respect to time yields, we get

$$
\dot{V}=S^{T} \widehat{J} \dot{S}+\frac{1}{2} S^{T} \dot{\hat{J}} S-\left(w^{*}-w\right)^{T} C^{T} \dot{w},
$$

with $U=u_{\mathrm{eq}}+u_{n}$ and simplifying

$$
\begin{aligned}
\dot{V}_{1}= & S^{T}(-\gamma \operatorname{sign}(S)+d)-\left(w^{*}-w\right)^{T} C^{T} \dot{w} \\
= & S^{T}(-\gamma \operatorname{sign}(S)+\widetilde{\gamma} \operatorname{sign}(S) \\
& \quad-\widetilde{\gamma} \operatorname{sign}(S)+d)-C^{T}\left(w^{*}-w\right)^{T} \dot{w} \\
\leq & -\left|S^{T}\right|\left(w^{T} \phi-w^{*^{T}} \phi+\varepsilon\right) \\
& -\left|S^{T}\right|(\widetilde{\gamma}-|d|)-\left|S^{T}\right|\left(w^{*}-w\right)^{T} \phi \\
= & -\sum_{i=1}^{3}\left|s_{i}\right| \cdot\left(\sum_{j=1}^{6}\left(w_{i j}-w_{i j}^{*}\right) h_{j}\right)
\end{aligned}
$$




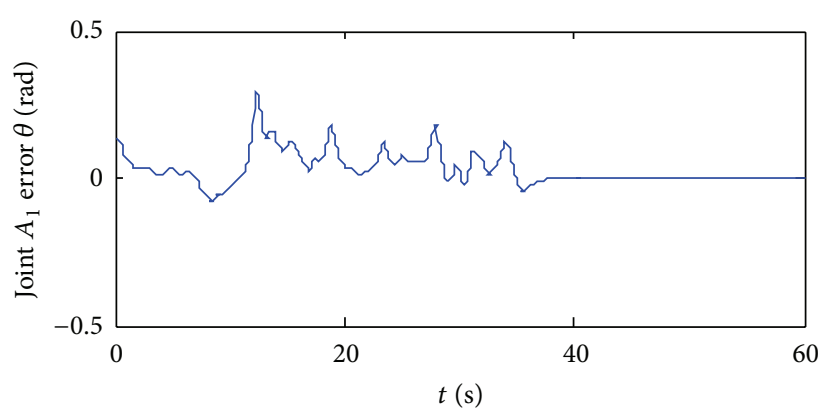

(a)

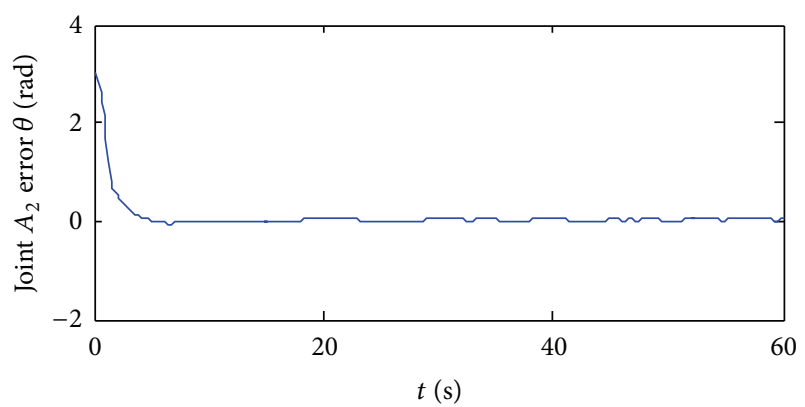

(b)

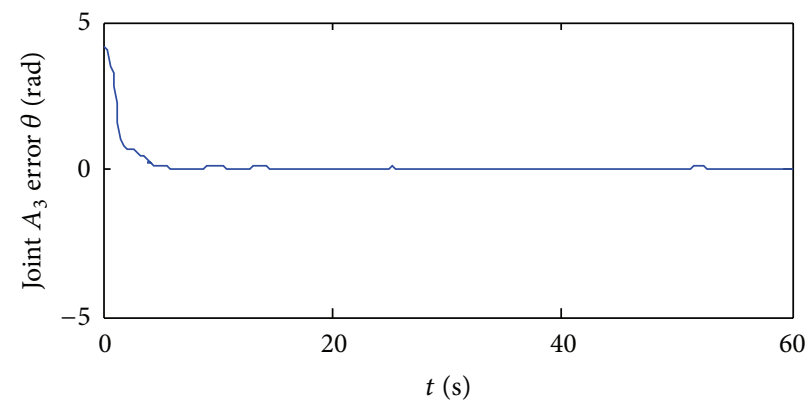

(c)

FIGURE 11: Angle displacement error responses controlled by TVSMC.

$$
\begin{aligned}
& -\left|S^{T}\right| \varepsilon-\left|S^{T}\right|(\tilde{\gamma}-|d|) \\
& -\sum_{i=1}^{3}\left|s_{i}\right|\left(\sum_{j=1}^{6}\left(w_{i j}-w_{i j}^{*}\right) h_{j}\right) \\
= & -\left|S^{T}\right| \varepsilon-\left|S^{T}\right|(\tilde{\gamma}-|d|) \\
\leq & \left|S^{T}\right| \varepsilon_{0}-\left|S^{T}\right| \varepsilon_{1} \\
= & -\left|S^{T}\right|\left(\varepsilon_{1}-\varepsilon_{0}\right) \leq 0 .
\end{aligned}
$$

Using integral transform in (19), the neural Lyapunov function can be gotten as

$$
\begin{aligned}
V_{1}(t) & =V_{1}(0)+\int_{0}^{t} \dot{V}_{1} d \tau \\
& \leq V_{1}(0)+\int_{0}^{t}-\left|S^{T}\right|\left(\varepsilon_{1}-\varepsilon_{0}\right) d \tau .
\end{aligned}
$$

By the Barbalat Lemma, the system is asymptotically stable.

\section{Simulation Tests}

The parameters of the 2-DOF parallel manipulator are listed as follows: joints reduction ratio is $40: 1, l_{11}=l_{12}=l_{21}=$ $l_{22}=l_{31}=l_{32}=244 \mathrm{~mm}$, back electromotive force constant is $0.04297 \mathrm{~V} /(\mathrm{s} / \mathrm{rad})$, torque constant is $3.41 \mathrm{~N} \cdot \mathrm{m} / \mathrm{A}$, resistance factor in driving side is $8.1 \times 10^{-5} \mathrm{~N} \cdot \mathrm{m} /(\mathrm{rad} / \mathrm{s})$, winding resistance of electrical machine is $1.025 \Omega$, winding inductance of electrical machine is $0.03837 \mathrm{H}$, rotational inertia $J$ is $0.39 \mathrm{~kg} \cdot \mathrm{m}^{2}$, and the uncertain inertia $\Delta J \leq$ $0.1 \mathrm{~kg} \cdot \mathrm{m}^{2}$. The desired manipulator trajectory is the right triangle shown in Figure 5, and the desired joints trajectories are shown in Figure 6.

The trajectory tracking processes controlled by the TVSMC algorithm and the RBF TVSMC algorithm are compared in Figures 7-12 with the corresponding angle displacement in Figure 6.

According to the angle displacement responses in Figures 7 and 8 , it can be seen that the maximum control voltage generated by TVSMC algorithms is $10 \mathrm{~V}$, while that by RBF TVSMC algorithms is no more than $2.5 \mathrm{~V}$. And the control voltage generated by the RBF TVSMC algorithms is more stable than the that by TVSMC algorithm. When we focus on the control accuracy, significant differences exist in steady-state regime of the closed-loop system as seen from the angle trajectory tracking responses in Figures 9 and 10. Figure 10 has more accurate trajectory tracking responses than Figure 9. According to angle displacement error responses of joint A1 in Figures 11 and 12, we can see that the angle error of joint stable at origin is within $40 \mathrm{~s}$ based on TVSMC algorithm, but based on RBF TVSMC algorithm it is not later than $3 \mathrm{~s}$. The average error responses controlled by the TVSMC algorithm are about $0.3 \mathrm{rad}$, while for the RBF SMC algorithm, the average error is less than $0.03 \mathrm{rad}$, which proves the precision improvement of the RBF TVSMC algorithm. 


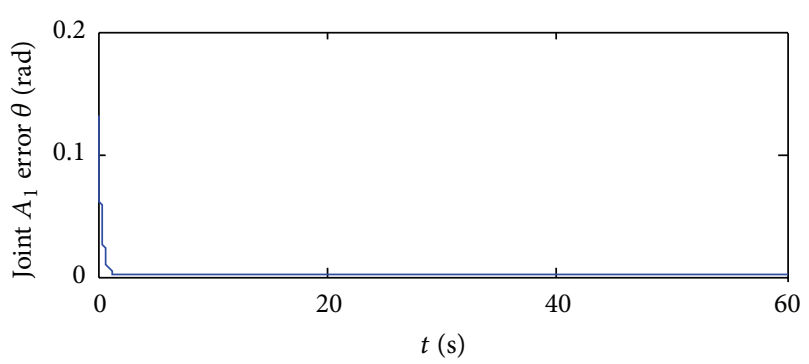

(a)

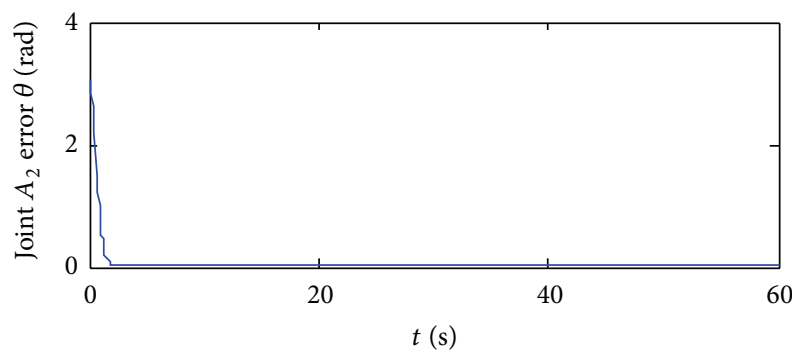

(b)

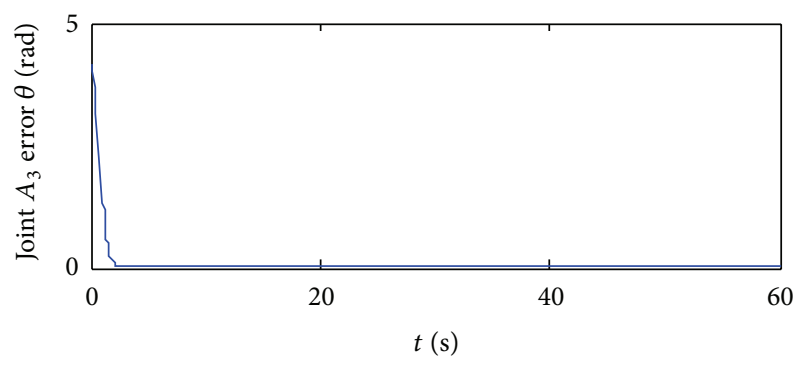

(c)

FIGURE 12: Angle displacement error responses controlled by RBF TVSMC.

\section{Conclusions}

In this paper, the accurate tracking control problem of 2-DOF parallel manipulator in the presence of parameter variation and uncertain disturbance is investigated via the TVSMC technique. An effective method is provided for the parameter selection in the TVSMC framework. RBF NN based time-varying sliding mode control algorithm is proposed to address the global chattering problem and increase the control accuracy. Simulation results verify the effectiveness of the proposed algorithm.

(1) This paper provides a trajectory algorithm of RBF neural network based time-varying sliding mode control for 2-DOF parallel manipulator system. And a compensator using RBF network is proposed to estimate the actuator nonlinearities and eliminate their upper boundaries. So that an RBF neural network controller can work properly requiring neither the evaluation of off-line dynamical model nor the time-consuming training process. The other article, "The Implementation to Servomotor Based on RBF Neural Network Equivalent to Sliding Mode Variable Structure Control," provides a trajectory optimization algorithm for 2-DOF parallel manipulator system of servomotor which is assumed as one linear system. The algorithm of input control is divided into two parts: one is the sliding mode control with the linear control and the other is the nonlinear control of output of the RBF replacing the switching input. (2) This paper is further research based on the other article, and it involves the three joints' trajectories but the other article just focuses on one joint.

\section{Acknowledgments}

The authors would like to thank the editors and the anonymous reviewers for their valuable comments and constructive suggestions. This work is supported by the National Natural Science Foundation of China (61201248) and Youth Foundation of JUST (KYY11081).

\section{References}

[1] M. R. Sirouspour and S. E. Salcudean, "Nonlinear control of hydraulic robots," IEEE Transactions on Robotics and Automation, vol. 17, no. 2, pp. 173-182, 2001.

[2] M.-L. Chan, C. W. Tao, and T.-T. Lee, "Sliding mode controller for linear systems with mismatched time-varying uncertainties," Journal of the Franklin Institute, vol. 337, no. 2-3, pp. 105$115,2000$.

[3] M. Rios-Bolívar, A. S. I. Zinober, and H. Sira-Ramírez, "Dynamical adaptive sliding mode output tracking control of a class of nonlinear systems," International Journal of Robust and Nonlinear Control, vol. 7, no. 4, pp. 387-405, 1997.

[4] S. A. Kowalchuk and C. D. Hall, "Spacecraft attitude sliding mode controller using reaction wheels," in AIAA/AAS Astrodynamics Specialist Conference and Exhibit, AIAA, Reston, Va, USA, August 2008.

[5] C. Li et al., "Improved neur-variable structure control of robot manipulators," Systems Engineering and Electronics, vol. 3, pp. 429-433, 2006.

[6] A. Blorfan, J. Mercklé, D. Flieller et al., "Sliding mode controller for three-phase hybrid active power filter with photovoltaic application," Smart Grid and Renewable Energy, vol. 3, no. 1, pp. 17-26, 2012.

[7] J. Y. Hung, W. Gao, and J. C. Hung, "Variable structure control: a survey," IEEE Transactions on Industrial Electronics, vol. 40, no. 1, pp. 2-22, 1993.

[8] Q. Khan, A. Iqbal, B. M. Iqbal et al., "Dynamic integral sliding mode control for SISO uncertain nonlinear systems," International Journal of Innovative Computing, Information and Control, vol. 8, no. 7, pp. 4621-4633, 2012.

[9] T.-C. Lin, S.-W. Chang, C.-H. Hsu et al., "Robust adaptive fuzzy sliding mode control for a class of uncertain discretetime nonlinear systems," International Journal of Innovative Computing, Information and Control, vol. 8, no. 1, pp. 347-359, 2012.

[10] L. Wu, W. X. Zheng, and H. Gao, "Dissipativity-based sliding mode control of switched stochastic systems," IEEE Transactions on Automatic Control, vol. 58, no. 3, pp. 785-793, 2013.

[11] L. Wu and D. W. C. Ho, "Sliding mode control of singular stochastic hybrid systems," Automatica, vol. 46, no. 4, pp. 779783, 2010.

[12] L. Wu, P. Shi, and H. Gao, "State estimation and sliding-mode control of markovian jump singular systems," IEEE Transactions on Automatic Control, vol. 55, no. 5, pp. 1213-1219, 2010. 
[13] F. Da, "Decentralized sliding mode adaptive controller design based on fuzzy neural networks for interconnected uncertain nonlinear systems," IEEE Transactions on Neural Networks, vol. 11, no. 6, pp. 1471-1480, 2000.

[14] J. Lin, R.-J. Lian, C.-N. Huang, and W.-T. Sie, "Enhanced fuzzy sliding mode controller for active suspension systems," Mechatronics, vol. 19, no. 7, pp. 1178-1190, 2009.

[15] P. C. Chen, C. W. Chen, and W. L. Chiang, "GA-based modified adaptive fuzzy sliding mode controller for nonlinear systems," Expert Systems with Applications, vol. 36, no. 3, pp. 5872-5879, 2009.

[16] C.-K. Lin, "Nonsingular terminal sliding mode control of robot manipulators using fuzzy wavelet networks," IEEE Transactions on Fuzzy Systems, vol. 14, no. 6, pp. 849-859, 2006.

[17] B. Cong, X. Liu, and Z. Chen, "Disturbance observer based time-varying sliding mode control for uncertain mechanical system," Journal of Systems Engineering and Electronics, vol. 23, no. 1, pp. 108-118, 2012.

[18] Z. Cao, Y. Zhao, and Q. Wu, "Adaptive trajectory tracking control for a nonholonomic mobile robot," Chinese Journal of Mechanical Engineering, vol. 24, no. 4, pp. 546-552, 2011.

[19] O. Barabones, P. Alkorta, and A. J. Garrido, "An adaptive sliding mode control scheme for induction motor drives," International Journal of Circuits, Systems and Signal Processing, vol. 1, pp. 7378, 2007.

[20] Y.-J. Huang, T.-C. Kuo, and S.-H. Chang, "Adaptive slidingmode control for nonlinear systems with uncertain parameters," IEEE Transactions on Systems, Man, and Cybernetics B, vol. 38, no. 2, pp. 534-539, 2008.

[21] J. Y. Hung, W. Gao, and J. C. Hung, "Variable structure control: a survey," IEEE Transactions on Industrial Electronics, vol. 40, pp. 2-21, 1993.

[22] C. Haizhong and G. guoqin, "The implementation to servomotor based on RBF neural network equivalent to sliding mode variable structure control," Small \& Special Electrical Machines, vol. 9, pp. 58-61, 2007.

[23] J. Angeles, Fundamentals of Robotic Mechanical Systems, Theory, Methods, and Algorithms, Mechanical Engineering Series, Springer, New York, NY, USA, 3rd edition, 2007.

[24] Y. Li, Dynamic Modeling and Control of a 2-DOF Redundantly Actuated Parallel Robot, Shandong University, 2010. 


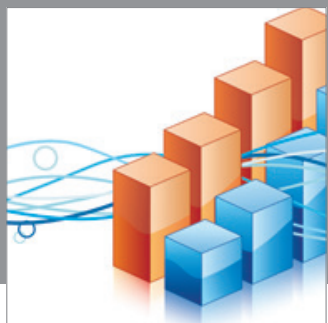

Advances in

Operations Research

mansans

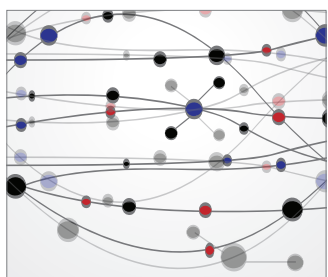

The Scientific World Journal
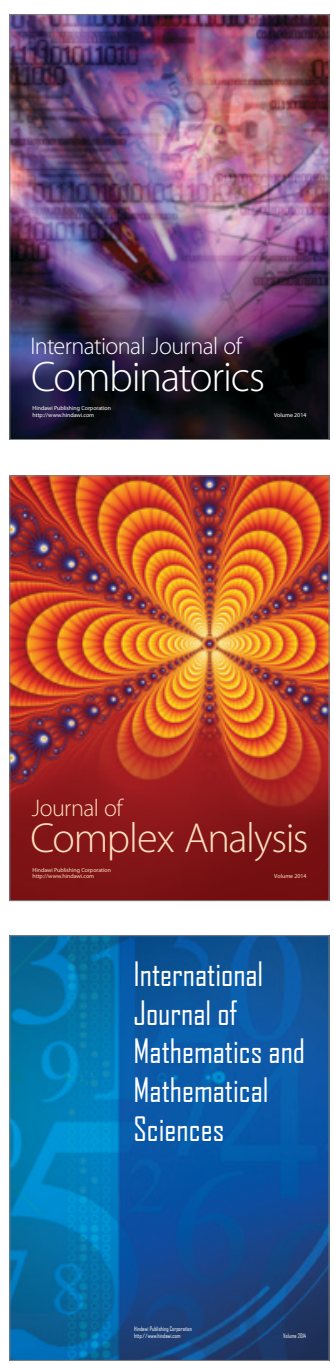
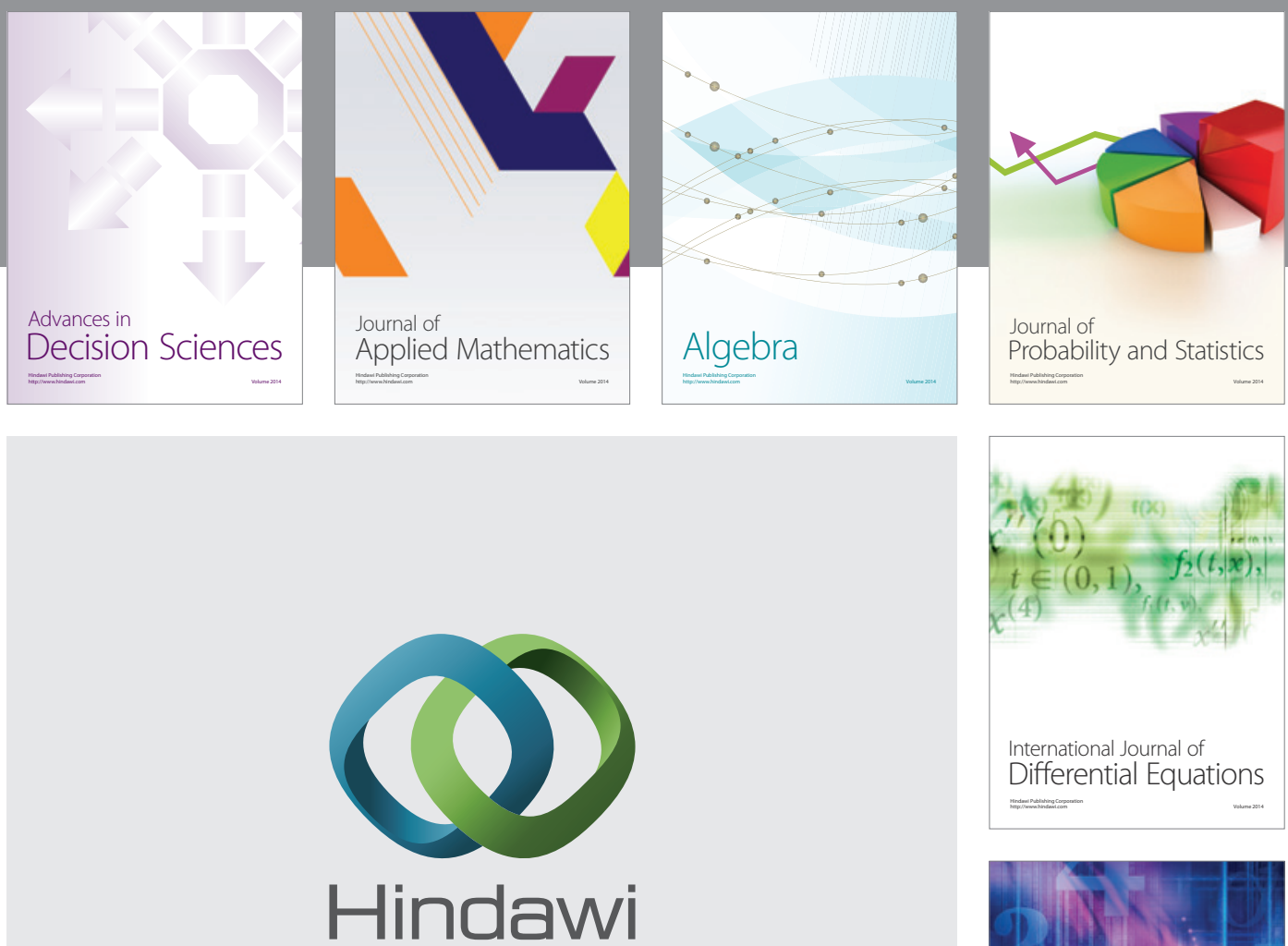

Submit your manuscripts at http://www.hindawi.com
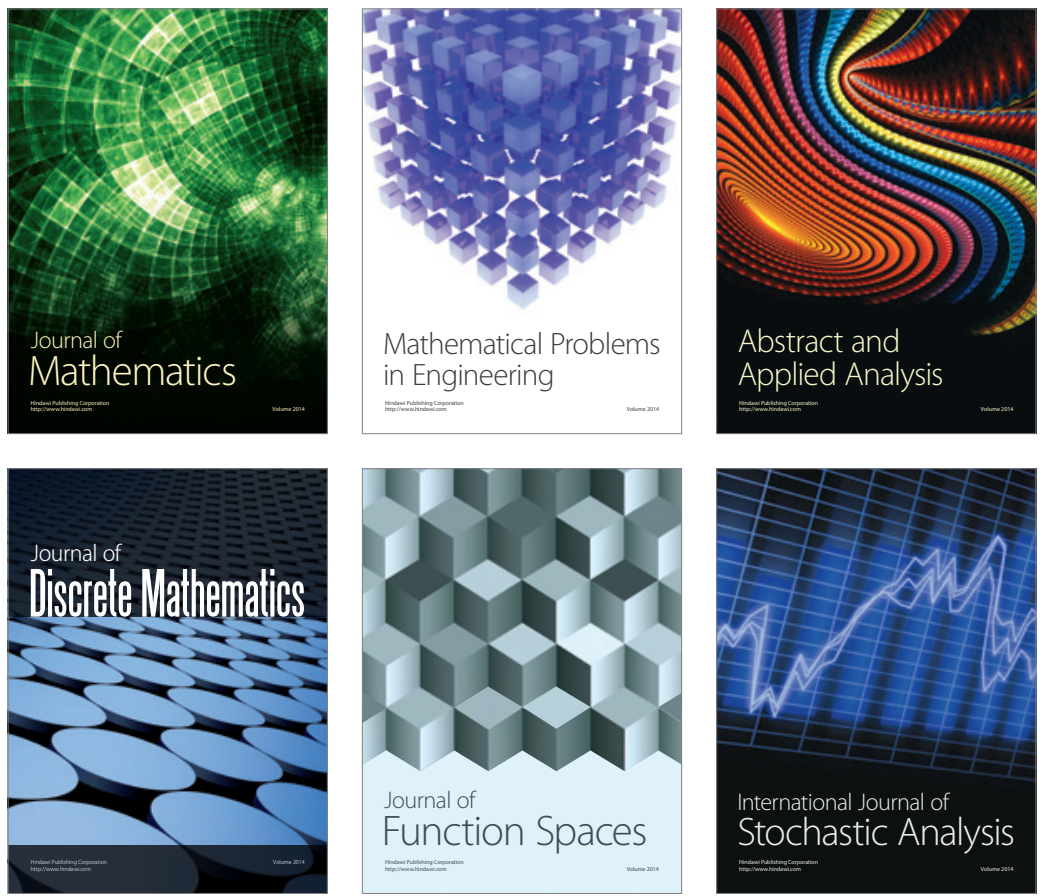

Journal of

Function Spaces

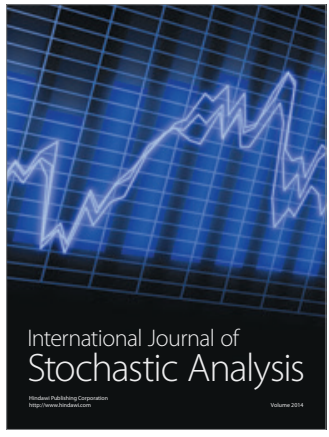

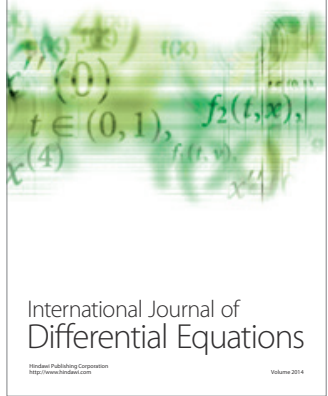
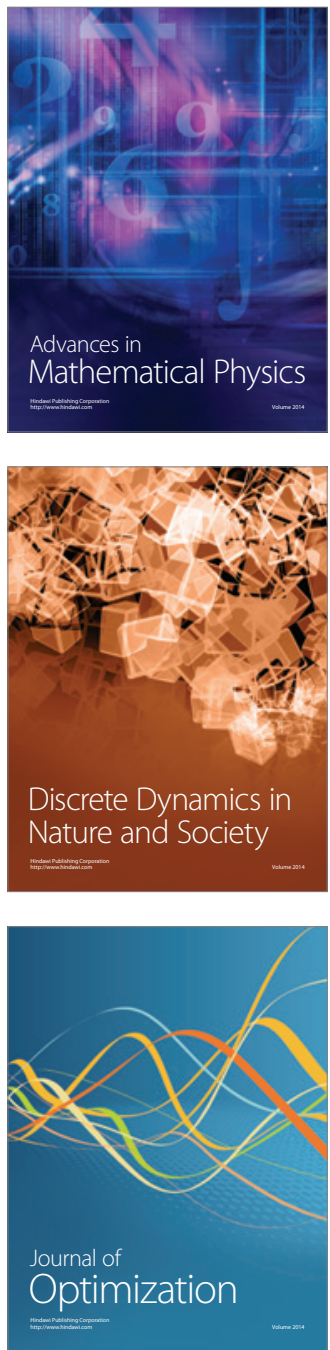